\title{
The early allergic response in small airways of human precision-cut lung slices
}

\author{
A. Wohlsen*, C. Martin*, E. Vollmer*, D. Branscheid", H. Magnussen", W-M. Becker*, \\ U. Lepp*, S. Uhlig*
}

The early allergic response in small airways of human precision-cut lung slices. A. Wohlsen, C. Martin, E. Vollmer, D. Branscheid, H. Magnussen, W-M. Becker, U. Lepp, S. Uhlig. C ERS Journals Ltd 2003.

ABSTRACT: To study the role of small airways in the early allergic response (EAR), the method of human precision-cut lung slices (PCLS) was developed and used to examine the bronchoconstriction elicited by passive sensitisation and allergen provocation.

Viable human PCLS of $250-\mu \mathrm{m}$ thickness containing airways $<1.5 \mathrm{~mm}$ in outer diameter were prepared from lung lobes obtained from lung resection and taken into culture. According to the low release of lactate dehydrogenase and the constant ciliary beat frequency, human PCLS were viable for at least 3 days.

Following overnight passive sensitisation with serum from allergic individuals, administration of grass-pollen extract or activating immunoglobulin $E$ antibody resulted in immediate airway contraction that was quantified by videomicroscopy. The extent of the EAR increased with decreasing airway size (outer airway diameter), with the strongest response occurring in the terminal bronchioles.

Histamine receptor antagonism was ineffective, and leukotriene or thromboxane receptor antagonism attenuated the early allergic response only in some cases. However, simultaneous blockade of leukotriene and thromboxane receptors almost completely prevented the early allergic response in the precision-cut lung slices from all individuals, suggesting such a dual treatment as a potential future asthma therapy. Eur Respir J 2003; 21: 1024-1032.
*Research Centre Borstel, Borstel and
${ }^{\#}$ Grosshansdorf Hospital, Centre for Pneumology and Thoracic Surgery, Grosshansdorf, Germany.

Correspondence: S. Uhlig, Research Centre Borstel, Division Pulmonary Pharmacology, Parkallee 22, D-23845 Germany.

Fax: 494537188778

E-mail: suhlig@fz-borstel.de

Keywords: Asthma

immunoglobulin $\mathrm{E}$

leukotrienes

small airways

thromboxane

Received: April 32002

Accepted after revision: February 122003

This study was supported by the Deutsche Forschungsgemeinschaft grant DFG Uh 88/3-1 to S. Uhlig.
Bronchoconstriction and airway hyperreactivity are major symptoms of asthma. The role of small airways $(<2 \mathrm{~mm}$ in diameter) in the disease process of bronchial asthma is still unresolved, but awareness of their importance is growing [1-3]. On the basis of measurements with the retrograde catheter technique in animals, MACKLEM and MEAD [4] determined that the small airways contribute $<10 \%$ of the total airway resistance and hence termed them the "quiet zone". More recently, however, using more sophisticated techniques, VAN BRABANDT et al. [5] suggested that peripheral resistance contributes between $50-90 \%$ of the total resistance in normal lungs and in the lungs of patients with chronic obstructive lung disease. Given that such a large fraction of airway resistance resides in the peripheral airways, it is not surprising that a number of clinical studies suggested peripheral airways as the predominant site of airflow obstruction in asthmatics [6-9]. Moreover, substantial airway inflammation is present in the peripheral airways of asthmatic patients $[3,10]$ and computational analyses based on quantitative histology led to the conclusion that the peripheral airways account for the majority of the airway hyperresponsiveness among these persons [11, 12]. Because airway inflammation and hyperresponsiveness are thought to be related to previous mast-cell degranulations during the early allergic response (EAR), responsiveness to allergens may, at least, partly explain these pathological alterations of small airway function and structure. However, with one exception, the contribution of small airways to the EAR has not been investigated in humans. Using sensitised bronchi, ElLIS et al. [13] showed that airways down to $0.5 \mathrm{~mm}$ in diameter are capable of an EAR. However, their method did not permit the authors to investigate airways of $<0.5 \mathrm{~mm}$ and to establish a correlation between airway size and the strength of the EAR.

In the past, the difficulty to perform functional studies with peripheral areas of the lung has hindered progress in this area of research. New insight into the small airways has become possible with the model of precision-cut lung slices (PCLS), which allows the analysis of the responses of airways down to the terminal bronchioles by quantitative videomicroscopy [14]. With this method, lung tissue, including airways, is cut into thin viable PCLS of $\sim 250 \mu \mathrm{m}$ thickness that are taken into culture. Functional parameters such as bronchoconstriction can be studied under a microscope and digital-imaging techniques make it possible to visualise and quantify contraction of airways. In contrast to conventional in-vitro methods of measuring bronchoconstriction, airway contraction in vivo is neither isometric nor isotonic, because in vivo the load that the muscle has to overcome increases as is shortens. Videomicroscopy of airways allows the determination of airway contraction and dilation under quasi-auxotonic conditions [15]. Another advantage of this method is that the total incubation volume is rather small $(0.5-1.0 \mathrm{~mL})$ so that the dilution of endogenously produced mediators is minimised.

Using rat PCLS, it has been shown previously that the EAR increases with decreasing airway size, being maximal at the terminal bronchioles [16]. However, asthma is difficult to model in experimental animals, for instance, serotonin, an 
important mast cell mediator of the EAR in rats [16], is almost absent from human mast cells [17]. The present study had three goals. First, to establish the method of PCLS for the measurement of bronchoconstriction and ciliary beat frequency in human lung tissue. Second, to use passively sensitised PCLS to investigate the significance of human small airways in the pathophysiology of asthma. Third, to use this novel model for pharmacological investigations of the EAR in human airways.

\section{Methods}

\section{Human precision-cut lung slices}

Human lung slices were prepared from patients undergoing surgery for cancer, most of them smokers. Carcinomas were later identified in the division of pathology as nonsmall cell carcinomas including squamous cell carcinoma and adenocarcinoma. Macroscopically healthy lung tissue at distance to the tumour was used to produce PCLS. The experiments were approved by the local ethics committee.

Macroscopically healthy areas were filled via the main bronchus with $500-800 \mathrm{~mL}$ low melting point agarose solution (final agarose concentration $1.5 \%$ ), and put on ice until the agarose had solidified. The used volume of agarose corresponds approximately to the total lung capacity (TLC) of this part of the lung [18]. An area distal to the tumour was then separated from the rest of the lobe, which remained with the pathologist for further examination. The lung tissue was cut further into 5-10 mm thick slabs from which cores along the airways were made with a coring tool. These cores, with a diameter of $0.9 \mathrm{~cm}$, were cut into $\sim 250 \mu \mathrm{m}$ thick slices by a Krumdieck tissue slicer (Alabama Research and Development, Munford, AL, USA). Subsequently, the PCLS were incubated in minimal essential medium (MEM; GIBCO Life Technologies, Eggenstein, Germany) and medium was changed every $30 \mathrm{~min}$ for the first $2 \mathrm{~h}$ and then every hour for the following $2 \mathrm{~h}$ to remove the agarose and mediators from the airways. Lung slices were cultured in medium based on MEM. The $10 \times$ concentrated MEM solution (w/o $\mathrm{NaHCO}_{3}$, w/o phenol red) was supplemented with amino acid solution (GIBCO), $200 \mathrm{nM}$ sodium pyruvate (GIBCO), vitamin solution (GIBCO), glucose (final concentration $2.2 \mathrm{mM}$ ), N-2hydroxyethylpiperazine- $N$-2-ethanesulphonic acid (HEPES) (4.6 mM) and $\mathrm{NaHCO}_{3}(5.2 \mathrm{mM})$. pH was adjusted to 7.4 before sterile filtration. Penicillin and streptomycin (GIBCO) were added to the MEM solution at a final concentration of $100 \mathrm{U} \cdot \mathrm{mL}^{-1}$ and $100 \mu \mathrm{g} \cdot \mathrm{mL}^{-1}$, respectively. For instillation of agarose, double-concentrated medium was mixed with the same volume of low melting point agarose solution at $37^{\circ} \mathrm{C}$. Slicing was performed in MEM buffer. MEM powder (without $\mathrm{NaHCO}_{3}$, with phenol red) with Earl's salts was supplemented with $6 \mathrm{~g} \cdot \mathrm{L}^{-1}$ HEPES and $2.2 \mathrm{~g} \cdot \mathrm{L}^{-1} \mathrm{NaHCO}_{3}$. The $\mathrm{pH}$ was adjusted to 7.4.

\section{Imaging and analysis}

The airways were imaged and digitised with a digital video camera as described earlier [16]. Airway area before addition of the allergen was defined as $100 \%$. Bronchoconstriction was expressed as the percentage decrease in airway area in comparison with the control airway area.

\section{Assessment of airway precontraction}

PCLS were cultured in MEM and the medium changed routinely to remove agarose and avoid effects of endogenously released mediators. Precontracted airways show a distinct folding of the airway epithelium and the portion of airway smooth muscle is $>50 \%$ of the outer airway area. From these observations, an experienced experimenter may estimate the degree of precontraction of airways. To verify this procedure, airways of 14 PCLS, which were not used for further experiments, were first assessed by the experimenter before they were treated with $10 \mu \mathrm{M}$ isoproterenol (Sigma, Taufkirchen, Germany) to obtain maximal dilation. The airway area and diameter was imaged before and after addition of the bronchodilator. Two of 14 PCLS were categorised as precontracted by the experimenter. Treatment with isoproterenol increased the median airway area to $117 \%$. In contrast, the two PCLS, categorised as precontracted, responded to isoproterenol by dilating to 170 and $345 \%$, respectively. From these findings and from other observations made during this study, the authors concluded that $\sim 10 \%$ of all airways were precontracted. Such airways were excluded from this study.

\section{Viability}

Viability of human PCLS was determined by measuring lactate dehydrogenase (LDH) leakage into the incubation medium and determining ciliary beat frequency over a time period of 3 days.

Lactate dehydrogenase leakage. Three PCLS per well were incubated in a 24-well plate and covered with $1 \mathrm{~mL}$ medium. At $0,12,14,36,48$, and $72 \mathrm{~h}$ slices were removed and put into $1 \mathrm{~mL} 0.2 \%$ Triton $\mathrm{X}-100$ and kept on ice until further processing. The supernatant was also removed and put on ice. PCLS samples were prepared for LDH measurement by tissue homogenisation in a Polytron tissue homogeniser (Kinematica AG, Littau, Switzerland). All samples, including the supernatant, were then centrifuged for $20 \mathrm{~min}$ at $11,000 \times \mathrm{g}$ at $4{ }^{\circ} \mathrm{C}$ to remove cell debris. Viability was expressed as the percentage LDH activity (Dimension pan; Dade Behring, Schwalbach, Germany) in the supernatant of the total LDH activity.

Ciliary beat frequency. Beating of cilia along the epithelial border of the airways was recorded with a fast camera (Visicam 640; Visitron Systems, Puchheim, Germany) recording 62 images per second at a magnification of 640. Along the epithelial border virtual lines were drawn representing 256 pixels. For each pixel, the change in light intensity was recorded and plotted against time. Ciliary beat frequency was determined by Fourier analysis. In each image measurements were obtained at three different sites of the luminal border. Such measurements were performed on three PCLS per patient. The data from each slice were averaged and the mean of the average reported.

Passive sensitisation and induction of the early allergic response. Human PCLS were treated overnight with $1 \%$ serum from patients with allergic rhinitis. Serum from three different patients with low specific immunoglobulin (Ig)E $\left(2.8 \mathrm{U} \cdot \mathrm{mL}^{-1}\right)$, medium specific $\operatorname{IgE}\left(50 \mathrm{U} \cdot \mathrm{mL}^{-1}\right)$ and high specific IgE $\left(420 \mathrm{U} \cdot \mathrm{mL}^{-1}\right)$ aged 35,50 and $28 \mathrm{yrs}$, respectively, was used. The specific IgE against timothy grass (Phleum pratense) pollen was quantified by fluorescent enzyme immunoassay (CAP-FEIA; Amersham Pharmacia, Freiburg, Germany). In addition, human PCLS were also treated with an activating goat antihuman $\operatorname{IgE}\left(100 \mathrm{ng} \cdot \mathrm{mL}^{-1}\right.$; Biosource, Nivelles, Belgium).

Single PCLS were incubated with $1 \mathrm{~mL}$ of serumcontaining $\mathrm{MEM}$ at $37^{\circ} \mathrm{C}$ and $5 \%$ carbon dioxide in an 
incubator overnight $(\sim 15 \mathrm{~h})$. The following day, the PCLS received fresh medium free of antibiotics and serum. Single slices were moved from the incubator onto a fresh 24-well plate, positioned within the well and then put under a microscope (Inverted microscopes Leica DMIRB and DMIL; Zeiss, Oberkochen, Germany). A control image was taken before addition of the allergen $\left(5 \mu \mathrm{g} \cdot \mathrm{mL}^{-1}\right.$ Phleum pollen extract), which was administered in prewarmed $\left(37^{\circ} \mathrm{C}\right) \mathrm{MEM}$. Image acquisition was started after addition of the solution. Images were made every $30 \mathrm{~s}$ for $10 \mathrm{~min}$.

\section{Pharmacological intervention studies}

To investigate the mediators of the EAR, PCLS were preincubated for $10 \mathrm{~min}$ with the leukotriene (LT)-receptor antagonist montelukast (MSD Sharp \& Dohme GmbH, Haar, Germany), the thromboxane-receptor antagonist SQ 29548 (SPI Bio, Paris, France) and the histamine (H)1receptor antagonist triprolidine, either alone or in combination prior to challenge with the allergen.

\section{Statistics}

The correlation analysis was performed by calculating the Spearman rank coefficient. The $\alpha$-error due to multiple comparisons was controlled by using the Bonferroni-Holm procedure.

\section{Results}

\section{The preparation of human precision-cut lung slices}

Preparation of human lung tissue yielded up to 30 slices from one resection with airways ranging from generation 12 $(\sim 1.5 \mathrm{~mm}$ in diameter [18]) down to the terminal bronchioles $(<0.6 \mathrm{~mm}$ in diameter). Viability of human PCLS was determined by measuring LDH leakage into the incubation medium and ciliary beat frequency over a time period of 3 days. $\mathrm{LDH}$ release remained $<10 \%$ for the first $24 \mathrm{~h}$ and did not exceed $15 \%$ within $72 \mathrm{~h}$ (fig. 1). In membranous airways of human PCLS, ciliary beat frequency ranged between 5-12 $\mathrm{Hz}$ at $37^{\circ} \mathrm{C}$, exhibiting only slight changes within $72 \mathrm{~h}$

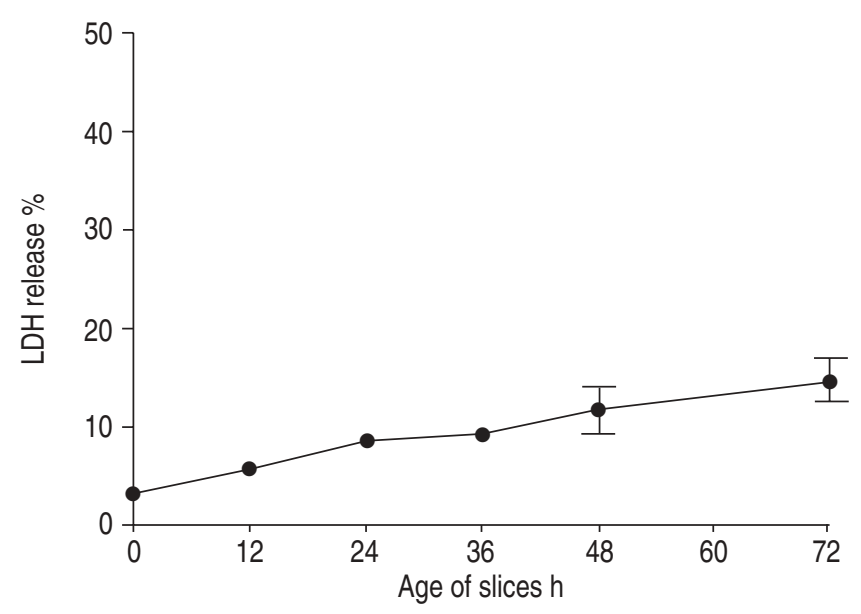

Fig. 1. - Leakage of lactate dehydrogenase (LDH) from human precisioncut lung slices over time. Lung slices were incubated in 24-well plates for up to $72 \mathrm{~h}$ in an incubator at $37^{\circ} \mathrm{C}$ and $5 \%$ carbon dioxide. Values shown are the mean \pm SD from three independent experiments.
Table 1. - Ciliary beat frequency in human precision-cut lung slices (PCLS) from individual lung donors determined on 3 consecutive days after preparation of the PCLS

\begin{tabular}{lrrrr}
\hline \multirow{2}{*}{ Patient } & \multicolumn{4}{c}{ Ciliary beat frequency $\mathrm{Hz}$} \\
\cline { 2 - 5 } & $24 \mathrm{~h}$ & $36 \mathrm{~h}$ & $72 \mathrm{~h}$ & Average \\
\hline 1 & 4.8 & 4.8 & 5.9 & 5.2 \\
2 & 9.1 & 9.0 & 9.3 & 9.1 \\
3 & 7.8 & 7.7 & 7.4 & 7.6 \\
4 & 6.1 & 8.8 & 7.2 & 7.4 \\
5 & 12.9 & 12.1 & 9.5 & 11.5 \\
\hline
\end{tabular}

(table 1). These findings demonstrate that human PCLS are viable for at least 3 days.

To further establish this model, the authors examined the responses of PCLS to acetylcholine and its stable analogue methacholine. Airways in human PCLS responded to acetylcholine by an immediate airway contraction that was reversible within $30 \mathrm{~min}$ (fig. 2a). The median effective extracellular concentration (EC50) for methacholine was $0.4 \mu \mathrm{M}$ (fig. 2b).
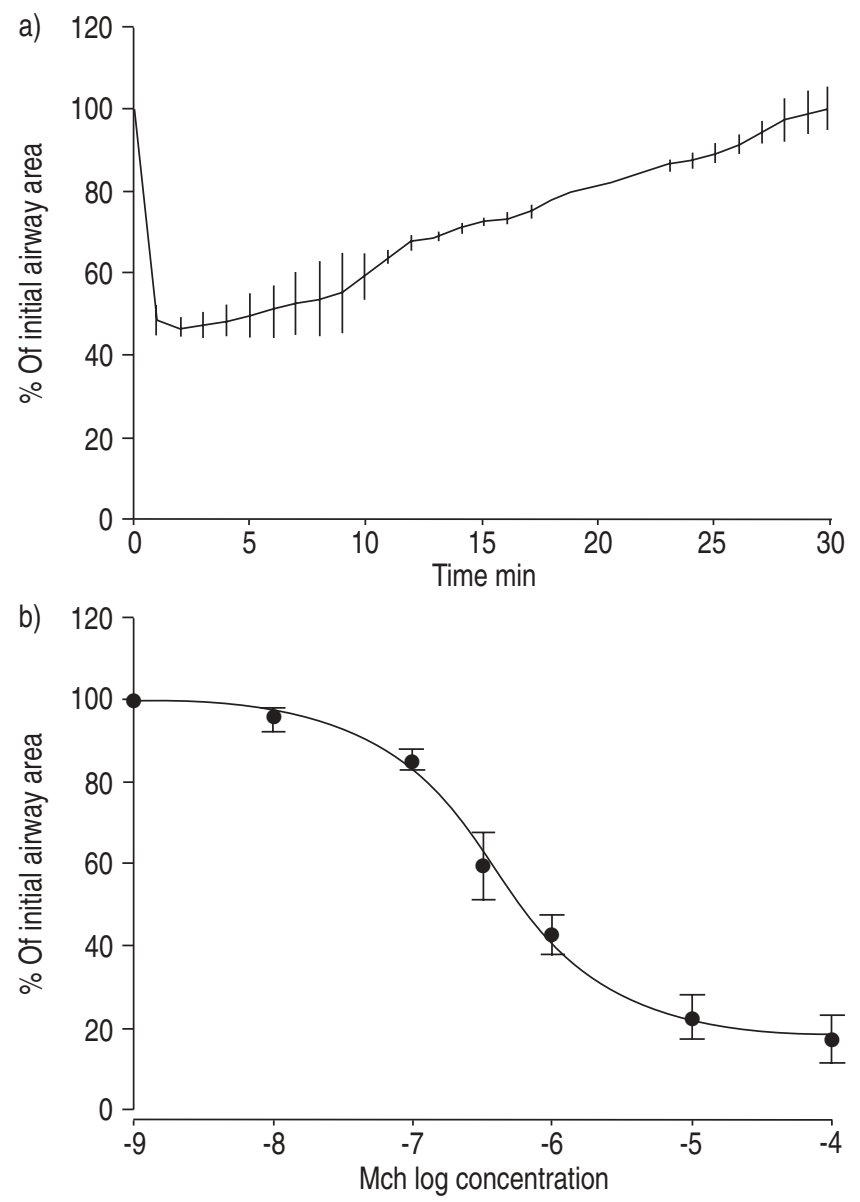

Fig. 2. - a) Time-dependency of acetylcholine (Ach) on airways of human precision-cut lung slices (PCLS) showing the contraction and dilation of the airways after exposure to Ach $\left(10^{-6} \mathrm{M}\right)$. Values are the mean \pm SD from PCLS of three different patients. b) Concentration/ response curve of methacholine (Mch) in small human PCLS. Mch was used for cumulative measurement at concentrations from $10^{-9}-10^{-4} \mathrm{M}$. Values shown are the mean \pm SEM from four to seven different patients. The medium airway size was $1.18 \mathrm{~mm}$ in outer airway diameter. 
The early allergic response

Human PCLS prepared from nonatopic patients were incubated overnight with sera from three different nonatopic patients containing high, medium and low concentratrions of IgE, specific for the pollen of timothy grass ( $P$. pratense). If slices were incubated with serum containing high or medium levels of specific IgE, airways contracted within 2 min of antigen challenge (figs 3 and 4). If, however, slices were incubated with serum from a nonatopic donor with a low level of specific IgE $\left(2.8 \mathrm{U} \cdot \mathrm{mL}^{-1}\right)$, addition of pollen extract had no effect (fig. 4). Furthermore, in nonsensitised PCLS, pollen extract caused no bronchoconstriction and a nonspecific antigen, such as ovalbumin, failed to induce bronchoconstriction in sensitised PCLS (data not shown). In contrast, incubation with an IgE-activating antibody caused contraction in sensitised PCLS (fig. 5). This indicates that the EAR in human PCLS is dependent on IgE.

\section{Effect of airway size on the early allergic response}

In PCLS, airway size can be measured and related to the responsiveness of each individual airway. Because human airways may have an inherent tone, which may affect the inner airway diameter, the authors used the outer airway diameter as a measure of airway size. In addition, airways that appeared to be precontracted were excluded from the analysis. Figure 6 shows a scatter diagram of the outer airway
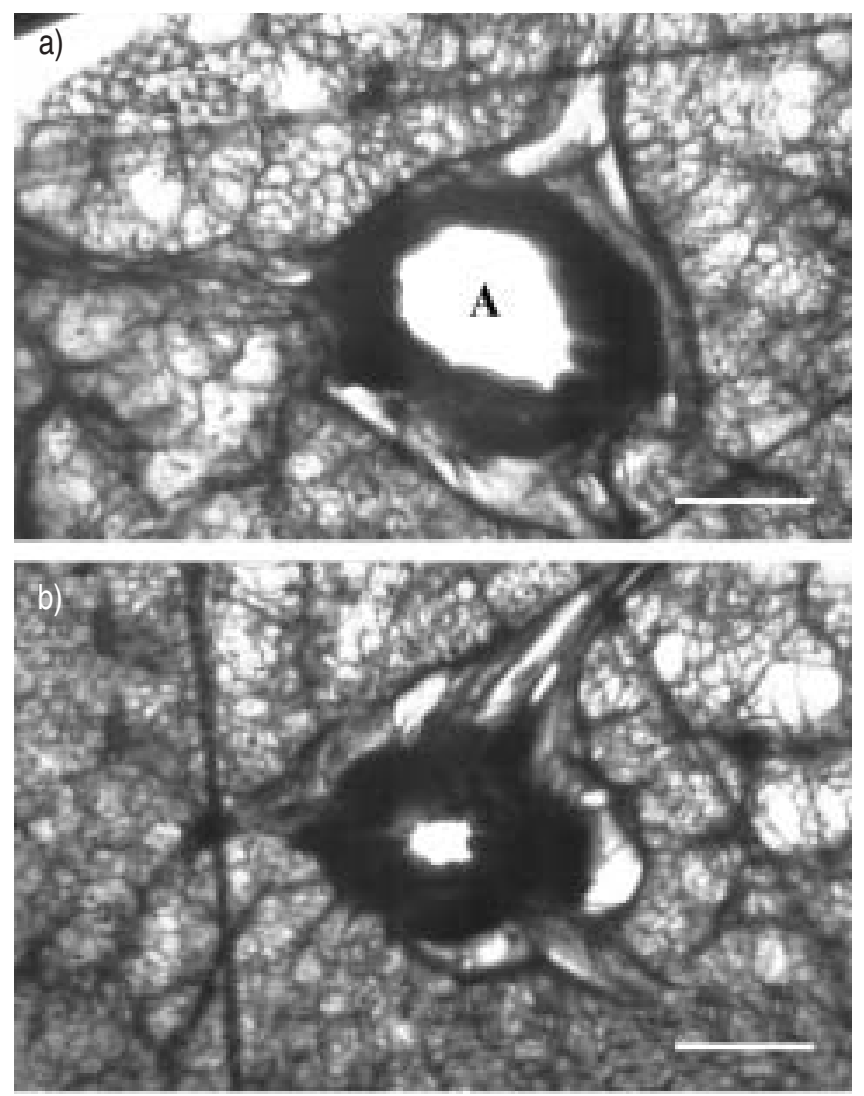

Fig. 3. - Images of a lung slice showing a single airway a) before and b) $10 \mathrm{~min}$ after administration of $5 \mu \mathrm{g} \cdot \mathrm{mL}^{-1}$ pollen extract. The human precision-cut lung slices were treated overnight with $1 \%$ serum from an atopic donor with a specific immunoglobulin-E titre $\left(50 \mathrm{U} \cdot \mathrm{mL}^{-1}\right)$ against timothy grass (Phleum pratense) pollen (passively sensitised). A: airway lumen. Internal scale bar=1 $\mathrm{mm}$.

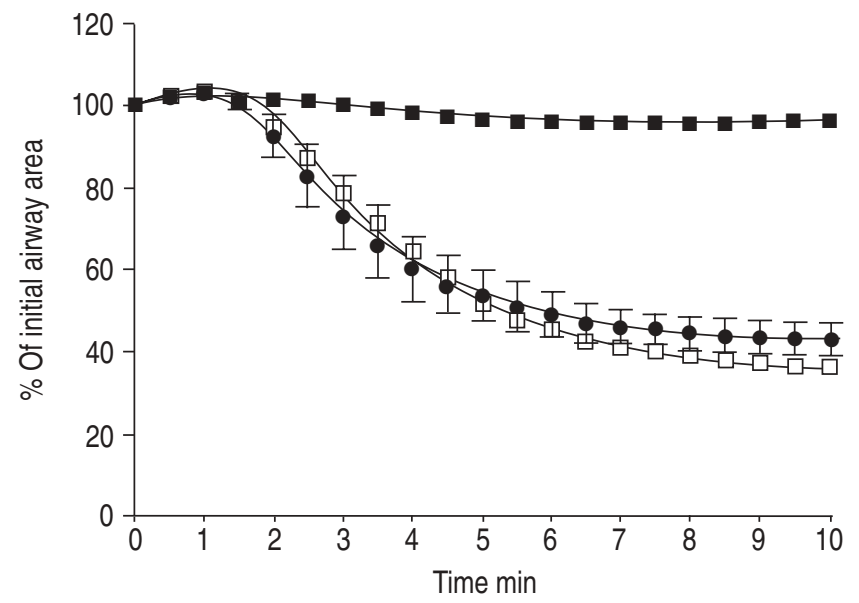

Fig. 4.-Time course of airway contraction in sensitised precision-cut lung slices (PCLS) after addition of $5 \mu \mathrm{g} \cdot \mathrm{mL}^{-1}$ pollen extract. Human PCLS were treated overnight with $1 \%$ serum from three atopic donors with low specific immunoglobulin $(\mathrm{Ig}) \mathrm{E}\left(2.8 \mathrm{U} \cdot \mathrm{mL}^{-1}, \mathrm{n}=4 ; \mathbf{\square}\right)$, medium $\operatorname{IgE}\left(50 \mathrm{U} \cdot \mathrm{mL}^{-1}, \mathrm{n}=16 ; \square\right)$ and high $\operatorname{IgE}\left(450 \mathrm{U} \cdot \mathrm{mL}^{-1}, \mathrm{n}=4 ; \bullet\right)$ titres against timothy grass (Phleum pratense). Values shown are the mean \pm SEM

diameter plotted against the maximum degree of airway contraction observed within $10 \mathrm{~min}$ of antigen challenge. A correlation was found between airway size and the extent of contraction $(\mathrm{r}=0.35, \mathrm{p}=0.0035)$, in that the strength of the antigen-induced contraction increased from larger airways to the terminal bronchioles.

Pharmacological characterisation of the early allergic response in human precision-cut lung slices

To identify the mediators responsible for inducing bronchoconstriction during the EAR, slices were preincubated with different inhibitors, either alone or in combination. Preincubation of passively sensitised PCLS with the serotonin-receptor antagonist ketanserin $(100 \mu \mathrm{M})$ before challenge did not affect the EAR (data not shown).

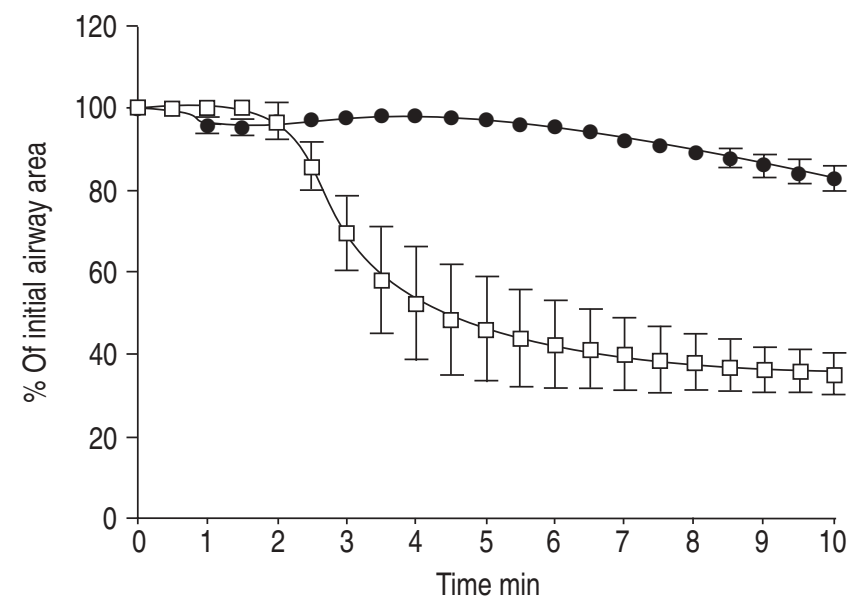

Fig. 5. - Time course of immunoglobulin (Ig)E-induced bronchoconstriction in sensitised and unsensitised precision-cut lung slices (PCLS). Human PCLS were incubated overnight with either a $1 \%$ serum high IgE titre $(\square)$ or with minimum essential medium only $(\bullet)$. Subsequently, the PCLS were treated with an activating anti-IgE antibody $\left(100 \mathrm{ng} \cdot \mathrm{mL}^{-1}\right)$. Values shown are the mean \pm SEM from PCLS obtained from three different lungs. 


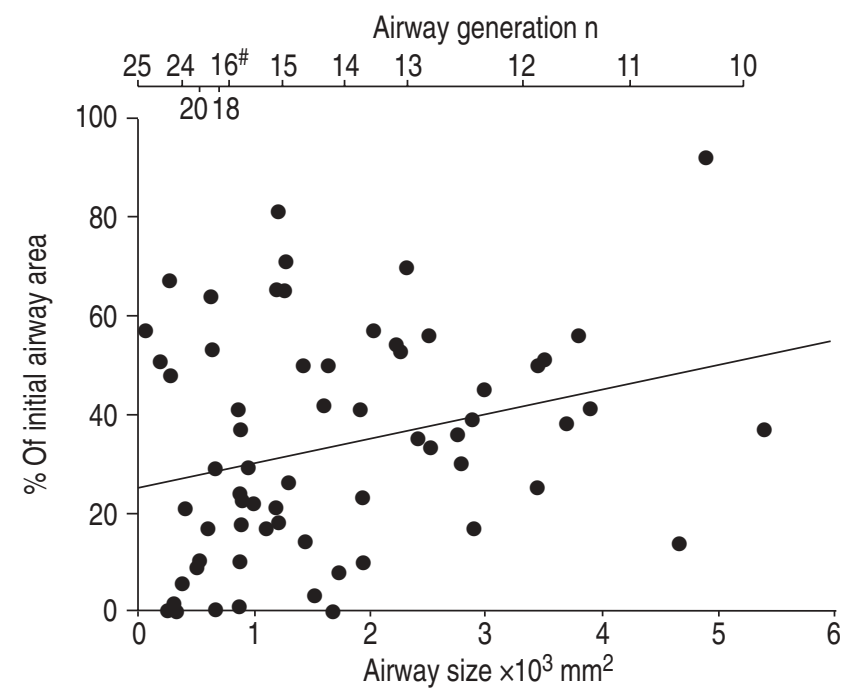

Fig. 6. - Correlation diagram of the extent of airway contraction induced by pollen extract in sensitised human precision-cut lung slices (PCLS) plotted against airway size (outer airway area). PCLS with airways of different sizes were passively sensitised overnight and subsequently challenged with $5 \mu \mathrm{g} \cdot \mathrm{mL}^{-1}$ pollen extract the next day. Each value represents the maximum contraction of a single airway with the given airway size. The top $\mathrm{x}$-axis indicates the corresponding outer airway generation according to YEH and SCHUM [18]. A total of 70 single lung slices from 25 nonatopic individuals (17 male, eight female, aged 42-77 yrs) were investigated. Data were analysed by calculating the Pearson correlation coefficient $(\mathrm{r}=0.35, \mathrm{p}=0.0035)$. \#: terminal bronchioles.

The individual responses of the PCLS from each of the 12 lung donors to pretreatment with the thromboxane-receptor antagonist SQ $29548(10 \mu \mathrm{M})$, the H1-receptor antagonist triprolidine $(5 \mu \mathrm{M})$ and/or the $\mathrm{LTD}_{4}$-receptor antagonist montelukast $(10 \mu \mathrm{M})$ are shown in figure 7 . All agents were used at a concentration at least 1,000-fold above their median inhibitory concentration values $[19,20]$. Bronchoconstriction was reduced in response to antigen challenge in three of 12 independent individual's PCLS pretreated with SQ 29548 (fig. 7) and in four of 12 pretreated with montelukast, whereas

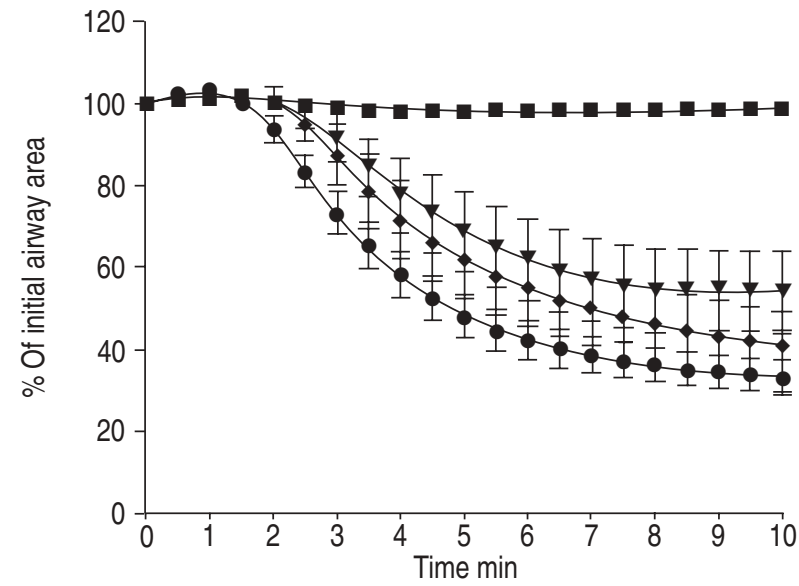

Fig. 8. - Time course of pollen extract-induced bronchoconstriction. Sensitised human precision-cut lung slices were treated with grass (Phleum pratense) pollen extract $(\bullet, \mathrm{n}=22)$ in the presence of montelukast $(\boldsymbol{\nabla}, \mathrm{n}=11)$, SQ $29465(\bullet, \mathrm{n}=15)$ or the combination of the two inhibitors $(\boldsymbol{\square}, \mathrm{n}=7)$. Values shown are the mean \pm SEM.

triprolidine appeared to be largely ineffective. Combination of triprolidine with either SQ 29548 or montelukast failed to attenuate the bronchoconstriction to allergen in PCLS. Of note, histamine-induced bronchoconstriction in human PCLS appeared normal with a maximal contraction of up to $40 \%$ of the initial airway area and an EC50 of $3 \mu \mathrm{M}\left(-\log _{10}\right.$ $\mathrm{EC} 50=5.5 \pm 0.14, \mathrm{n}=5)$. The combination of SQ 29548 and montelukast largely prevented the EAR in all cases (12 of 12). The kinetics of the allergen-induced bronchoconstriction were comparable in slices treated with SQ 29548 or montelukast (fig. 8).

As experimental animal studies have suggested that LTs exhibit their action via the formation of thromboxane [21], lung slices were preincubated with SQ 29548 to block thromboxane receptors and subsequently challenged with $\mathrm{LTC}_{4}$ or $\mathrm{LTD}_{4}$. Administration of $\mathrm{LTC}_{4}$ and $\mathrm{LTD}_{4}$ induced bronchoconstriction, despite pretreatment with SQ 29548 (table 2). The authors therefore conclude that in human lungs, LTs exert bronchoconstriction independently from thromboxane.
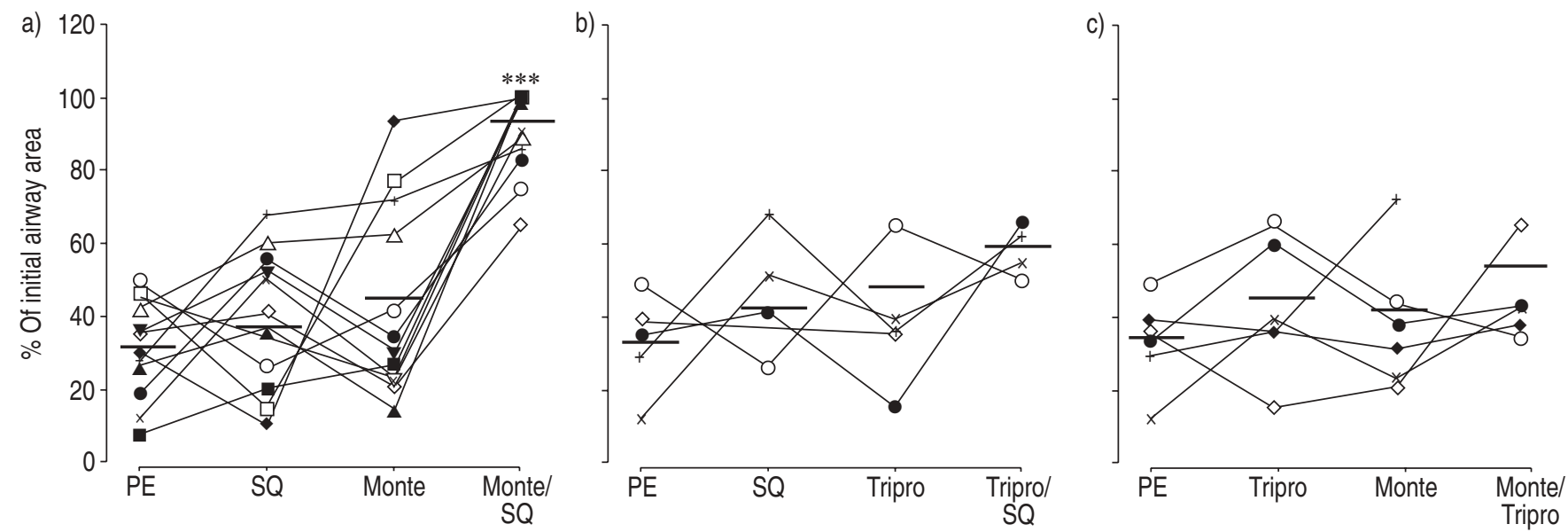

Fig. 7. - Effect of cysteinyl-leukotriene-, histamine 1-, and/or thromboxane-receptor blockade on the early allergic response in human precisioncut lung slices (PCLS). PCLS were passively sensitised overnight and subsequently challenged with $5 \mu \mathrm{g} \cdot \mathrm{mL}^{-1}$ pollen extract the next day. Ten minutes before allergen challenge, airways were pretreated with $10 \mu \mathrm{M}$ montelukast (Monte), $10 \mu \mathrm{M}$ SQ 29548 (SQ) or $5 \mu \mathrm{M}$ triprolidine (Tripro). Shown are a) the combination of Monte and SQ, b) the combination of Tripro and SQ and c) the combination of Monte and Tripro. The data represent slices from 13 different nonatopic individuals (eight females, five males, mean age 58.6 yrs). Medians are shown as horizontal lines. **: $\mathrm{p}<0.01$ versus pollen extract (PE) alone. 
Table 2.-Leukotriene (LT)-induced bronchoconstriction in human precision-cut lung slices (PCLS)

\begin{tabular}{lccc}
\hline Agonist & Pretreatment & Airway area \% of initial area & n \\
\hline LTC $_{4}$ & & $37.5 \pm 12.7$ & 4 \\
LTC $_{4}$ & SQ 29548 & $33.9 \pm 17.4$ & 4 \\
LTD $_{4}$ & & $29.1 \pm 24.7$ & 3 \\
LTD $_{4}$ & SQ 29548 & $30.1 \pm 35.9$ & 3 \\
\hline
\end{tabular}

Data are presented as mean $\pm \mathrm{SD}$ from $\mathrm{n}$ independent experiments Effect of $\mathrm{LTC}_{4}(10 \mathrm{nM})$ or $\mathrm{LTD}_{4}(10 \mathrm{nM})$ in the presence or absence of the thromboxane receptor antagonist SQ $29548(10 \mu \mathrm{M})$ on airway area in human PCLS.

\section{Discussion}

Asthma is characterised by airway obstruction, inflammation of the airway mucosa, and airway hyperresponsiveness. The initial step of the allergic airway obstruction is the EAR. In this study, the focus was placed on the response in small airways. The data suggest that the EAR can take place in small human airways and may be treated effectively by dual administration of LT- and thromboxane-receptor antagonists.

\section{The model of precision-cut lung slices}

Because of the possibility to examine airways of all airway generations with diameters $<5 \mathrm{~mm}$, PCLS are suited to investigate the responsiveness of individual airways in relation to their size. To this end, single slices are monitored by videomicroscopy, and airway size, airway contraction, vessel contraction and ciliary beat frequency are determined by digital-imaging techniques. Taking advantage of this method, the authors have recently defined the responsiveness of small airways to methacholine [14], thromboxane [22], endothelin [22] and allergen [16] in rats. Until now, human PCLS have been used in toxicological studies [23], but not to investigate the responses of single human airways. As judged from the release of LDH and the constant ciliary beat frequency, human PCLS were viable for $\geqslant 72 \mathrm{~h}$. The present observations match those made previously, which indicated that PCLS are viable for $\geqslant 1-3$ days [14, 23].

There are several potential problems in the use of PCLS to measure small airway responses. 1) Are airways in PCLS precontracted and how would this affect the measurements? 2) Is there load on airway smooth muscle? 3) Does instillation of agarose and inflation to TLC change airway responses? 4) Is the use of airway area instead of the diameter adequate for the analysis of bronchoconstriction?

In response to these questions: 1) all PCLS with strong visible precontraction were excluded from this study. Thus, although it cannot be completely ruled out that some airways were precontracted, it may be assumed that the extent of precontraction in the PCLS that were used in this study was only small. Furthermore, to exclude any effect of precontraction, in the correlation analysis the authors used the outer rather than the inner airway diameter. Finally, similar results to those reported here were obtained in airways of rats [16], which lack an inherent airway tone [14]. 2) Lung recoil resides primarily in the lung parenchyma which is under some tension even at low lung volumes [24]. As small airways are directly connected to the parenchyma this is expected to put some load on the airway smooth muscle in small airways with an intact surrounding microanatomy as in PCLS. However, at present there is no data on whether airway smooth muscle in PCLS is loaded or not. Nonetheless, given the fact that the microanatomy is maintained and that the PCLS reopen once the stimulus is removed (fig. 2) [16], the authors believe that airway smooth muscle is loaded at least to some extent in PCLS. Certainly, load builds up once the airways start to contract and the surrounding tissue is distorted. The load that builds up during contraction may be enhanced by the fact that the lung tissue is held in place by the weight of a platinum wire as described [14]. Furthermore, if lack of load would significantly alter airway responses in PCLS, the airways in PCLS would be expected to contract more readily than in the intact lung. However, as the authors have shown previously, for various mediators, the median effective concentrations required for airway contraction and increases in airway resistance are the same in PCLS and in intact lungs, respectively [25]. 3) The preparation of PCLS requires that lungs be filled with high volumes of agarose approaching TLC [18]. ADLER et al. [26] investigated the elasticity of air-filled lungs in contrast to agarose-filled lungs and found no difference in the elasticity of the parenchyma in their preparation. Another concern is that the high pulmonary pressures of up to $30 \mathrm{cmH}_{2} \mathrm{O}$, used for filling the lungs with agarose, may alter recoil and tethering forces as well as the parenchyma-airway interdependence. DANDURAND et al. [27] investigated the effect of different agarose filling volumes on airway area and methacholine responsiveness in razor bladecut rat lung slices. They observed that increasing lung volume resulted in increased airway area, but had no effect on maximal airway narrowing (if comparing filling with 50 or $75-100 \%$ TLC, although a small effect between 25-100\% TLC was reported) or the EC50. Thus, filling the lungs at TLC appears to increase the size of airways, but not their responsiveness. In addition, because these authors did not report the outer airway diameter, it cannot be excluded that at the lower filling volumes the airways were not fully recruited. Finally, these authors did not find any differences related to airway size. 4) With respect to the calculation of airway responses, it may be asked whether utilisation of the airway area will not exaggerate any possible role of small airways, because any change in area corresponds at least to a square change in radius. However, according to Poiseulle's law the determinant of airflow is the fourth power of the radius, suggesting that airway area is a better approximation than airway radius. This is also supported by the excellent correlation between EC50 values obtained from area measurements in PCLS and from measurements of pulmonary resistance in isolated perfused lungs [25]. Moreover, the relative changes remain identical regardless of whether area or radius is used. For instance, if in figure 6 the correlation coefficient is calculated by using outer airway diameter instead of area, the same value for the nonparametric Spearman correlation coefficient $(0.32, \mathrm{p}=0.0075)$ is obtained, and only slightly different values if Pearson's (parametric) correlation coefficient is calculated $(0.35, \mathrm{p}=0.0035$ based on diameter versus $0.34, \mathrm{p}=0.0051$ based on area).

Taken together, measurement of airway area in PCLS appears to be a reliable tool to study small airway responses. EC50 values obtained with PCLS or with conventional methods are in the same order. For example, the EC50 of $3 \mu \mathrm{M}$ for histamine-induced bronchoconstriction in human PCLS matches that in human tracheal preparations [28]. The EC50 value for methacholine-induced bronchoconstriction in human PCLS was $0.4 \mu \mathrm{M}$ compared with $1 \mu \mathrm{M}$ in isolated human bronchi [29]. This difference may be explained by the observation from rat PCLS that smaller airways are more sensitive to methacholine [14].

\section{Airway size and the early allergic response}

Small airways have been defined as those with a diameter of $<2 \mathrm{~mm}[1,2]$. As a result of their relative inaccessibility, their 
role in human asthma has only been poorly defined. In the present study, the authors demonstrate that PCLS offer a novel approach to study bronchoconstriction in small human airways. While these findings clearly demonstrate that sensitised small airways in PCLS respond to allergen, it is difficult to predict whether these observations also reflect the ability of small airways in intact human lungs to respond to inhaled stimuli. As noted above, contractile responses of airways of any size are a complex process depending on the forces exerted by the surrounding tissue and on the load on the airway smooth muscle. Nevertheless, these findings are in line with and extend those clinical and experimental findings summarised above, which all suggest an important role of small airways in asthma. In addition, at least in the case of thromboxane, the greater sensitivity of small airways within PCLS [22] is confirmed by histological analysis of intact lungs [30]. Finally, some clinical studies have shown superior efficacy on asthma symptoms of aerosols with improved delivery to the lung periphery [31-33].

Extrapolation to the situation of human asthmatics is also complicated by the poor understanding of inhaled allergen deposition. Upon inhalation the relatively large pollen particles $(20-30 \mu \mathrm{m})$ will most probably not immediately reach the lung periphery, but it has been suggested that they may agglomerate in the small airways independently from daily pollen exposure, as a result of the gradual cumulative effect of deposition of small amounts of allergen in the lower airways [34]. More recently, however, it was demonstrated that grass pollen allergens might form aerosols with particle sizes between $0.12-4.67 \mu \mathrm{m}$ [35], small enough to reach the small airways. It also seems possible that therapeutic strategies, which preferentially open larger airways, may help to channel allergens to the smaller airways, thereby shifting symptoms towards the lung periphery.

With all these reservations in mind, the regression analysis shown in figure 4 nonetheless indicates that the EAR became stronger with decreasing airway size, as the slope of the regression line between airway size and the extent of bronchoconstriction was significantly different from zero, although this effect was less pronounced in human compared with rat PCLS [16]. Why small airways, at least in PCLS, exhibit a stronger response to allergen is unknown. They appear not to be more sensitive in general, as is exemplified by endothelin-1, to which all airway generations respond similarly [22]. A possible explanation would be a heterogeneous distribution of mast cells, which are mainly responsible for the mediator release during the EAR [36, 37]. In fact, based on lung wet weight, allergen-challenged peripheral lung tissue releases more prostanoids, LTs and histamine than bronchial tissue $[13,38]$. Alternatively, a larger percentage of unoccupied $\mathrm{IgE}$ receptors on mast cells in peripheral airways than central airways might lead to a facilitated degranulation of these mast cells [13]. A third possibility is that cysteinyl (Cys)LT $\mathrm{L}_{1}$ and thromboxane prostanoid (TP) receptors, both of which have been identified in great numbers in the human lung [39-41], are heterogeneously distributed throughout the bronchial tree. Finally, structural differences between large bronchi and small bronchioles, such as the relatively greater muscular thickness in peripheral airways might account for differences in airway reactivity $[42,43]$.

Hence, smooth muscle shortening may result in greater narrowing of smaller airways [44]. As a note of caution, most of the lung tissue used for the preparation of PCLS was obtained from smokers. Since smoking of $>10$ packs per year can lead to peripheral airway inflammation and increased airway responsiveness [45], the authors cannot exclude effects on smooth muscle shortening by smoking habits.

\section{Mediators of the early allergic response}

The authors have no direct evidence for the presence of mast cells in PCLS, but it is generally accepted that the EAR is caused by IgE-dependent mast cell activation [17]. While the role of mast cells was not addressed in the present study, the essential role of $\mathrm{IgE}$ for the sensitisation of lung tissue was confirmed by the dependency on IgE in the serum (fig. 4) and by the bronchoconstriction induced by an IgE-activating antibody (fig. 5). Upon triggering, pulmonary human mast cells release a variety of different mediators, among them bronchoconstrictors such as histamine, LTs, prostaglandin $\mathrm{D}_{2}$ and thromboxane [17]. These mediators cause airway contraction by binding to specific receptors on airway smooth muscle, i.e. histamine to $\mathrm{H} 1$ receptors, LTs to CysLT receptors and both prostaglandin $\mathrm{D}_{2}$ and thromboxane to TP receptors [46, 47]. Previously, ELLIS et al. [13] demonstrated in isolated human bronchi that the combined triple inhibition of prostanoids, LTs and histamine largely prevented allergen-induced bronchoconstriction in small and large human airways. However, they made no attempt to further identify the specific roles of these mediators. In the present study, the authors extended these observations by investigating the effect of all possible one-way and two-way combinations of H1-, CysLT- and TP-receptor antagonists. They found that the H1-receptor antagonist triprolidine was ineffective, and CysLT- or TP-receptor blockade was effective only in some cases. These findings are in line with no proven and documented benefit of antihistamines in asthma [48], and with the clinical observations of CysLT1- and TP-receptor antagonists being effective only in subsets of patients [46-51]. Notably, however, simultaneous blockade of CysLT- and TPreceptors provided nearly complete protection from antigeninduced airway contraction in PCLS from all individuals. The mechanistic basis for this synergism is unknown, but unlike in guinea-pigs [52] and rats [53], in humans, LTs do not exhibit their action via the secondary release of thromboxane [54] (table 2). These findings suggest that in humans the EAR is mediated by a synergistic effect of TP-receptor agonists and peptido-LTs, at least in the small airways. To the best of the authors' knowledge, with the exception of a recent single case study [55], in humans the cooperative effects between a CysLT-receptor antagonist and a TP-receptor antagonist have not been described before.

The authors conclude that precision-cut lung slices offer a useful way to study physiological responses in human lung tissue. Using this novel method, they have shown that an early allergic response is principally possible even in very small human airways. Acknowledging some reservations as to possible differences between small airways in situ and in precision-cut lung slices, their findings further suggest that the early allergic response may become stronger with decreasing airway size. Finally, these data indicate that simultaneous targeting of leukotriene and thromboxane receptors in small airways may be considered as a novel treatment in asthma.

Acknowledgements. The perfect technical assistance of C. Rodde and D. Buchholz is gratefully acknowledged.

\section{References}

1. Howarth PH. The role of small airways in asthma: a review and key questions. Am J Respir Crit Care Med 1998; 157: S173-S207. 
2. Kraft M. The distal airways: are they important in asthma? Eur Respir J 1999; 14: 1403-1417.

3. Tulic MK, Christodoulopoulos P, Hamid Q. Small airways inflammation in asthma. Respir Res 2001; 2: 333-339.

4. Macklem P, Mead J. Resistance of central and peripheral airways measured by a retrograde catheter. J Appl Physiol 1967; 22: 395-401.

5. van Brabandt $\mathrm{H}$, Cauberghs $\mathrm{M}$, Verbeken $\mathrm{E}$, Moerman $\mathrm{P}$, Lauweryns JM, van de Woestijne KP. Partitioning of pulmonary impedance in excised human and canine lungs. J Appl Physiol 1983; 55: 1733-1742.

6. Ingram RHJ. Physiological assessment of inflammation in the peripheral lung of asthmatic patients. Lung 1990; 168: 237-247.

7. Wagner EM, Bleeker ER, Permutt S, Liu MC. Direct assessment of small airways reactivity in human subjects. Am J Respir Crit Care Med 1998; 157: 447-452.

8. Yanai M, Sekizawa K, Ohrui T, Sasaki H, Takishima T. Site of airway obstruction on pulmonary disease: direct measurement of intrabronchial pressure. J Appl Physiol 1992; 72: 1016-1023.

9. Wagner EM, Liu MC, Weinman GG, Permutt S, Bleeker ER. Peripheral lung resistance in normal and asthmatic subjects. Am Rev Respir Dis 1990; 141: 584-588.

10. Roche WR. Inflammatory and structural changes in the small airways in bronchial asthma. Am J Respir Crit Care Med 1998; 157: S191-S194.

11. Wiggs BR, Bosken CH, Pare PD, James AL, Hogg JC. A model of airway narrowing in asthma and in chronic obstructive pulmonary disease. Am Rev Respir Dis 1992; 145: 1251-1258.

12. Kuwano K, Bosken $\mathrm{CH}$, Pare PD, Bai TR, Wiggs ER, Hogg JC. Small airway dimensions in asthma and in chronic obstructive pulmonary disease. Am Rev Respir Dis 1993; 148: $1220-1225$.

13. Ellis JL, Hubbard WC, Meeker S, Undem BJ. Ragweed antigen $\mathrm{E}$ and anti-IgE in human central versus peripheral bronchi. Am J Respir Crit Care Med 1994; 150: 717-723.

14. Martin C, Uhlig S, Ullrich V. Videomicroscopy of methacholine-induced contraction of individual airways in precision-cut lung slices. Eur Respir $J$ 1996; 9: 24792487.

15. Schmidt DT, Dent G, Ruhlmann E, Munoz NM, Leff AR, Rabe KF. Studying human airway pharmacology in microsections: application of videomicrometry. Eur Respir J 2002; 19: 991-996.

16. Wohlsen A, Uhlig S, Martin C. Immediate allergic response in small airways. Am J Respir Crit Care Med 2001; 163 : 1462-1469.

17. Galli SJ, Costa JJ. Mast cells. In: Crystal RG, West JB, Barnes PJ, Weibel ER, The Lung: Scientific Foundations. 2nd Edn. Philadelphia, Lipincott-Raven Publishers, 1997; pp. 929-946.

18. Yeh HC, Schum GM. Models of human lung airways and their application to inhaled particle deposition. Bull Math Biol 1980; 42: 461-480.

19. Jones TR, Labelle M, Belley M, et al. Pharmacology of montelukast sodium (Singulair), a potent and selective leukotriene $\mathrm{D}_{4}$ receptor antagonist. Can J Physiol Pharmacol 1995; 73: 191-201.

20. Takano $\mathrm{S}$, Ishimoto $\mathrm{H}$, Nakahata $\mathrm{N}$, Nakanishi $\mathrm{H}$. Thromboxane A2 receptor characterization in human astrocytoma cells and rabbit platelets by a new thromboxane antagonist, [3H]ONO NT-126. Res Commun Chem Pathol Pharmacol 1992; 76: 155-170.

21. Fujimura M, Bando T, Mizuhashi K, Matsuda T. Interaction of thromboxane A2 and leukotrienes in guinea pig airways in vivo. Prostaglandins 1991; 42: 379-389.

22. Martin C, Ullrich V, Uhlig S. Effects of the thromboxane receptor agonist U46619 and endothelin-1 on large and small airways. Eur Respir J 2001; 16: 316-323.

23. Fisher RL, Smith MS, Hasal SJ, Hasal KS, Gandolfi AJ,
Brendel K. The use of human lung slices in toxicology. Hum Exp Toxicol 1994; 13: 466-471.

24. Hoppin FG Jr. Parenchymal mechanics and asthma. Chest 1995; 107: 140S-144S.

25. Held HD, Martin C, Uhlig S. Characterization of airway and vascular responses in murine lungs. Br J Pharmacol 1999; 126: 1191-1199.

26. Adler A, Cowley EA, Bates JH, Eidelman DH. Airwayparenchymal interdependence after airway contraction in rat lung explants. J Appl Physiol 1998; 85: 231-237.

27. Dandurand RJ, Wang CG, Phillips NC, Eidelman DH. Responsiveness of individual airways to methacholine in adult rat lung explants. $J$ Appl Physiol 1993; 75: 364-372.

28. Bai TR. Abnormalities in airway smooth muscle in fatal asthma. Am Rev Respir Dis 1990; 141: 552-557.

29. Jongejan RC, de Jongste JC, Raatgeep RC, Stijnen T, Bonta IL, Kerrebijn KF. Effects of inflammatory mediators on the responsiveness of isolated human airways to methacholine. Am Rev Respir Dis 1990; 142: 1129-1132.

30. Uhlig S, Nüsing R, von Bethmann A, et al. Cyclooxygenase2-dependent bronchoconstriction in perfused rat lungs exposed to endotoxin. Mol Med 1996; 2: 373-383.

31. Rees PJ, Clark TJ, Moren F. The importance of particle size in response to inhaled bronchodilators. Eur J Respir Dis 1982; 119: 73-78.

32. Busse WW, Brazinsky S, Jacobson K, et al. Efficacy response of inhaled beclomethasone dipropionate in asthma is proportional to dose and is improved by formulation with a new propellant. J Allergy Clin Immunol 1999; 104: 12151222.

33. Magnussen H. Equivalent asthma control after dose reduction with HFA-134a beclomethasone solution aerosol. Comparative Inhaled Steroid Investigation Group (CISIG). Respir Med 2000; 94: 549-555.

34. Driessen MN, Quanjer PH. Pollen deposition in intrathoracic airways. Eur Respir J 1991; 4: 359-363.

35. Taylor PE, Flagan RC, Valenta R, Glovsky MM. Release of allergens as respirable aerosols: A link between grass pollen and asthma. J Allergy Clin Immunol 2002; 109: 51-56.

36. Nagase T, Moretto A, Dallaire MJ, Eidelman DH, Martin JG, Ludwig MS. Airway and tissue responses to antigen challenge in sensitized brown Norway rats. Am J Respir Crit Care Med 1994; 150: 218-226.

37. Bachelet CM, Bernaudin JF, Fleury-Feith J. Distribution and histochemical characterization of pulmonary mast cells in the rat and guinea pig. Int Arch Allergy Appl Immunol 1988; 87: 225-229.

38. Schulman ES, Adkinson NF, Newball HH. Cyclooxygenase metabolites in human lung anaphylaxis: airway vs. parenchyma. J Appl Physiol 1982; 53: 589-595.

39. Lynch KR, O'Neill GP, Liu Q, et al. Characterization of the human cysteinyl leukotriene CysLT1 receptor. Nature 1999; 399: 789-793.

40. Norman P, Cuthbert NJ, McKenniff MG, Gardiner PJ. The thromboxane receptors of rat and guinea-pig lung. Eur J Pharmacol 1992; 229: 171-178.

41. Namba T, Sugimoto Y, Hirata M, et al. Mouse thromboxane A2 receptor: cDNA cloning, expression and northern blot analysis. Biochem Biophys Res Commun 1992; 184: 1197-1203.

42. Ebina M, Yaegashi H, Chiba R, Takahashi T, Motomiya M, Tanemura M. Hyperreactive site in the airway tree of asthmatic patients revealed by thickening of bronchial muscles. A morphometric study. Am Rev Respir Dis 1992; 141: $1327-1332$.

43. Moreno RH, Hogg JC, Pare PD. Mechanics of airway narrowing. Am Rev Respir Dis 1986; 133: 1171-1180.

44. Mitchell HW, Cvetkovski R, Sparrow MP, Gray PR, McFawn PK. Concurrent measurement of smooth muscle shortening, lumen narrowing and flow to acetylcholine in large and small porcine bronchi. Eur Respir J 1998; 12: 10531061 . 
45. Mullen M, Wiggs BR, Wright JL, Hogg JC, Pare PD. Nonspecific reactivity in cigarette smokers. Am Rev Respir Dis 1986; 133: 120-125.

46. Beasley RCW, Featherstone RL, Church MK, et al. Effect of a thromboxane receptor antagonist on $\mathrm{PGD}_{2}$ - and allergeninduced bronchoconstriction. J Appl Physiol 1989; 66: 16851693.

47. Magnussen H, Boerger S, Templin K, Baunack AR. Effects of a thromboxane-receptor antagonist, BAY u 3405, on prostaglandin $\mathrm{D}_{2}$ - and exercise-induced bronchoconstriction. J Allergy Clin Immunol 1992; 89: 1119-1126.

48. Rabe KF, Schmidt DT. Pharmacological treatment of asthma today. Eur Respir J 2000; 16: Suppl. 34, 34 40s.

49. Drazen JM, Silverman EK, Lee TH. Heterogeneity of therapeutic responses in asthma. $\mathrm{Br}$ Med Bull 2000; 56: 1054-1070.

50. Aizawa H, Shigyo M, Nogami H, Hirose T, Hara N. BAY u3405, a thromboxane A2 antagonist, reduces bronchial hyperresponsiveness in asthmatics. Chest 1996; 109: 338-342.

51. Tanifuji Y, Goto S, Yoshida $\mathrm{T}$, et al. The effect of
anti-TXA2 receptor antagonist on the asthmatic subject and characterization of responder. Eur Respir J 1999; 14: Suppl. 30, 287s.

52. Yamaguchi T, Mukaiyama O, Itoh K, Satoh Y, Terada A Iizuka Y. Effects of CS-518, a thromboxane synthase inhibitor, on the asthmatic response. Eur $J$ Pharmacol 1993; 236: 43-50.

53. Uhlig S, Wendel A. Lipid mediators in perfused rat lung. In: Von Wichert P, Siegenthaler S, eds. Interdisziplinäre Aspekte der Pneumologie [Interdisciplinary Aspects in Pneumology] Stuttgart, Thieme Verlag, 1995; pp. 66-74.

54. Gorenne I, Norel X, Brink C. Cysteinyl leukotriene receptors in the human lung: what's new? Trends Pharmacol Sci 1996; 17: $342-345$

55. Takahashi N, Ishibashi $\mathrm{Y}$, Murakami $\mathrm{Y}$, et al. Beneficial effect of combination therapy with ozagrel and pranlukast in exercise-induced asthma demonstrated by krypton- $81 \mathrm{~m}$ ventilation scintigraphy a case report. Ann Acad Med Singapore 2000; 29: 766-769. 